\title{
LIST OF THE INTERNATIONAL REVIEWERS IN 2019
}

The Korean Journal of Orthodontics enjoyed another great successful year in 2019. Hundreds of well-prepared manuscripts have been submitted from many countries for publication and, in reviewing of those manuscripts, our orthodontic experts have contributed in various ways to maintain a higher standard of the Journal. Among them, we hopes to extend our special thanks to the following international reviewers invited in 2019. Their expertise, dedication, and uncompromising demand for scientific sophistication were all instrumental to assuring the high quality expected by our readers.

Abdolreza Jamilian, Iran

Abhishek Singha Roy, India

Ahmad S Burhan, Syrian Arab Republic

Alberto Caprioglio, Italy

Alessandra Lucchese, Italy

Ali Merdji, Algeria

André Machado, Brazil

Andrea Fonseca Jardim da Motta, Brazil

Andrea Scribante, Italy

Anis Farhan Kamaruddin, Malaysia

Arbel Sharan, Israel

Bancha Samruajbenjakun, Thailand

Beatriz Tarazona-Alvarez, Spain

Benedict Wilmes, Germany

Bong-Kuen Cha, Korea

Bum-Soon Lim, Korea

Cahide Aglarci, Turkey

Carlo Fornaini, France

Carlos Flores-Mir, Canada

Carole Charavet, Belgium

Cesar de Oliveira, United Kingdom

Cheol-Hyun Moon, Korea

Chiarella Sforza, Italy

Chihiro Arai, Japan

Chooryung Chung, Korea

Christoph Bourauel, Germany

Chung How Kau, United States

Chung-Chen Jane Yao, Taiwan

David A. Covel, United States

Demetrios Halazonetis, Greece

Denize Ramirez de Souza, Brazil

Derya Germec Cakan, Turkey

Ding Bai, China

Do-Gyoon Kim, United States

Dong Hwa Chung, Korea

Dong-Soon Choi, Korea

DongYul Lee, Korea

Eduardo Espinar -Escalona, Spain

Eiji Tanaka, Japan
Ellen Wen-Ching Ko, Taiwan

Eric Liou, Taiwan

Eung-Kwon Pae, United States

EZGI ATIK, Turkey

Fatma Deniz Uzuner, Turkey

Fazal Shahid, Malaysia

Fidan Alakuş Sabuncu, Turkey

Flavio Mendonca Copello, Brazil

Flavio Uribe, United States

Furkan Dindaroğlu, Turkey

Giulio Alessandri Bonetti, Italy

H. W. Fields, United States

Hande Gorucu Coskuner, Turkey

Hee-Moon Kyung, Korea

Heloísa Helena Filipe Alves Proença, Japan

Henrik Lund, Sweden

Heon Jae Cho, Korea

Hiroaki Takeda, Japan

Hiroshi Kamioka, Japan

Hoi-Jeong Lim, Korea

Hooman Zarif Najafi, Iran

Hwi-Dong Jung, Korea

Hyo-Sang Park, Korea

Hyo-Won Ahn, Korea

Hyung Jun Kim, Korea

Hyung Seog Yu, Korea

Il-Hyung Yang, Korea

Il-Sik Cho, Korea

Insan Jang, Korea

Jae Hyun Park, United States

Jae Sung Kwon, Korea

Jagan K Baskaradoss, India

Janghoon Ahn, Korea

Jeong-Ho Choi, Korea

Ji-Hoi Moon, Korea

Ji-Hwan Kim, Korea

Jin Hyoung Cho, Korea

Ji-Yeon Lee, Korea

Joe Antoun, New Zealand
Joe Rebellato, United States

Jong Ghee Kim, Korea

Jong-Moon Chae, Korea

Jong-Wan Kim, Korea

Joong-Ki Kook, Korea

Josef Freudenthaler, Austria

JS Lee, United States

Jung-Hoon Kim, Korea

Jung-Sub An, Korea

Jung-Yul Cha, Korea

Junji Sugawara, Japan

Kee-Joon Lee, Korea

Ki Beom Kim, United States

KiChul Tae, Korea

Ki-Ho Park, Korea

Koray Onur Sanal, Turkey

Kwangchul Choy, Korea

Kyung A Kim, Korea

Kyung Hwa Kang, Korea

Kyung-Ho Kim, Korea

Laura Roduiguez-Vilc, Mexico

Leonardo Tavares Camardella, Brazil

Leslie A. Will, United States

Lorenzo Franchi, Italy

Lorne D. Koroluk, United States

M. Ali Darendeliler, Australia

Maike Holm, Germany

Manuel Lagravere, Canada

Marcos Rogerio Mendonca, Brazil

Maria Joao Ponces, Portugal

ME Devereux, Ireland

Meenakshi Vishwanath, United States

Mehmet Okan Akcam, Turkey

Mihee Hong, Korea

Min-Ho Jung, Korea

Mohamed I Masoud, United States

Mohamed T Ellabban, Egypt

Mohammad Khursheed Alam, Malaysia

MS Ravi, India 
Muge Aksu, Turkey

My Huy Thuc Le, Malaysia

Nam-Ki Lee, Korea

Natalia Zamora, Spain

Na-young Chang, Korea

Nelson Gerald, United States

Neslihan Ebru Şenışık, Turkey

Nita Viwattanatipa, Thailand

Noriaki Yoshida, Japan

Nuria Esther Gallardo-Lopez, Spain

Oral S, Turkey

Özge Çelik Güler, Turkey

Paolo M. Cattaneo, Denmark

Paula Mendes Santos, Brazil

Philip E Benson, United Kingdom

Prakash Kharbanda, India

Rafael Scaf de Molon, Brazil

Raffaele Spena, Italy

Renato Bigliazzi, Brazil

Renske Hiemstra, The Netherlands

Rizomar Ramos do Nascimento, Brazil

Rosaria Bucci, Italy

Ryo Hamanaka, Japan

Saidi Jaafar, Malaysia

Sang Jin Sung, Korea

Sang-Cheol Kim, Korea

Sang-Min Lee, Korea

Sankalp Sood, India
Satpal S Sandhu, India

Saulo Gabriel Moreira Falci, Brazil

Seok-Ki Jung, Korea

Seong Ho Han, Korea

Seong Sik Kim, Korea

Seong-Gon Kim, Korea

Seong-Hun Kim, Korea

Sercan Akyalcin, United States

Seung Pyo Lee, Korea

Seung-Hak Baek, Korea

Seung-Ho Ohk, Korea

Seung-Youp Lee, Korea

Shouichi Miyawaki, Japan

Shuai Cao, Chile

Siti Adibah Othman, Malaysia

Sohaib Shujaat, Belgium

Soon Shin Hwang, Korea

Soon-Man Kwon, Korea

Spyridon N. Papageorgiou, Germany

Sug-Joon Ahn, Korea

Su-Jung Kim, Korea

Sung Jin Kim, Korea

Sung-Hoon Lim, Korea

Sung-Hwan Choi, Korea

Sung-Nam Gang, Korea

Sung-Seo Mo, Korea

Sun-Hyung Park, Korea

Tae-Woo Kim, Korea
Takashi Ono, Japan

Takashi Yamashiro, Japan

Theodore Eliades, Switzerland

Thyagaseely Premaraj, United States

Till Edward Bechtold, Germany

Timm Cornelius Schott, Germany

Toru Deguchi, United States

Wan Nurazreena Wan Hassan, Malaysia

Wey Mang Chek, Malaysia

Won Hee Lim, Korea

Woo-Sung Son, Korea

Xianglong Han, China

Yasuhiro Shimizu, Japan

Yong-Il Kim, Korea

Yong-Keun Lee, Korea

Yong-Suk Choi, Korea

Yong-Taek Jung, Korea

Yoon Jeong Choi, Korea

Yoon-Ah Kook, Korea

Yoon-Goo Kang, Korea

Yoonji Kim, Korea

Yoon-Ji Kim, Korea

Yoon-Ji Kim, Korea

Yoshiro Matsumoto, Japan

Yu Li, China

Zuisei Kanno, Japan 